\title{
Rectifying Electrical Synapses Can Affect the Influence of Synaptic Modulation on Output Pattern Robustness
}

\author{
Gabrielle J. Gutierrez and Eve Marder \\ Volen Center for Complex Systems and Biology Department, Brandeis University, Waltham, Massachusetts 02454
}

Rectifying electrical synapses are commonplace, but surprisingly little is known about how rectification alters the dynamics of neuronal networks. In this study, we use computational models to investigate how rectifying electrical synapses change the behavior of a small neuronal network that exhibits complex rhythmic output patterns. We begin with an electrically coupled circuit of three oscillatory neurons with different starting frequencies, and subsequently add two additional neurons and inhibitory chemical synapses. The five-cell model represents a pattern-generating neuronal network with two simultaneous rhythms competing for the recruitment of a hub neuron. We compare four different configurations of rectifying synapse placement and polarity, and we investigate how rectification changes the functional output of this network. Rectification can have a striking effect on the network's sensitivity to alterations of the strengths of the chemical synapses in the network. For some configurations, the rectification makes the circuit dynamics remarkably robust against changes in synaptic strength compared with the nonrectifying case. Based on our findings, we predict that modulation of rectifying electrical synapses could have functional consequences for the neuronal circuits that express them.

\section{Introduction}

The classical study demonstrating the electrical synapse in the crayfish escape circuit also showed that the connection was rectifying, that is positive current flows preferentially in one direction (Furshpan and Potter, 1959). Subsequently, the importance of electrical coupling in both invertebrate and vertebrate nervous systems has become evident (White et al., 1986; Rela and Szczupak, 2004; Pereda et al., 2013; Sasaki et al., 2013). Electrical synapses are often thought to promote speed and synchrony in excitable tissues, such as the heart and in the nervous system. Although network synchronization often results from electrical coupling (Chow and Kopell, 2000; Lewis and Rinzel, 2000; Pfeuty et al., 2003), gap junctions can have the opposite effect by desynchronizing network neurons. For example, electrical coupling between Golgi neurons produces surround inhibition when neurons receive sparse excitatory inputs (Vervaeke et al., 2010). Furthermore, electrical synapses create complex input-output functions in combination with chemical synapses (Spira et al., 1976; Kopell and Ermentrout, 2004; Rela and Szczupak, 2004).

The present study aims to further our understanding of electrical synapses by exploring their rectification in a neuronal circuit model. A single cell can express multiple gap-junction monomers, thus forming a diverse set of gap junction hemichannels (Dermietzel, 1998). Rectifying gap junctions are

Received March 1, 2013; revised June 27, 2013; accepted July 4, 2013.

Author contributions: G.J.G. and E.M. designed research; G.J.G. performed research; G.J.G. analyzed data; G.J.G. and E.M. wrote the paper.

This work was supported by the National Institutes of Health Grants NS17813 and MH46742.

The authors declare no competing financial interests.

Correspondence should be addressed to Eve Marder, Volen Center MS 013, Brandeis University, 415 South Street, Waltham, MA 02454.E-mail: marder@brandeis.edu.

DOI:10.1523/JNEUROSCI.0937-13.2013

Copyright $\odot 2013$ the authors $\quad 0270-6474 / 13 / 3313238-11 \$ 15.00 / 0$ thought to result from the joining of two different gap junction hemi-channels (Phelan et al., 2008).

Because junctions are known to be rectifying only when electrophysiological, intracellular recordings have been made (Devor and Yarom, 2002), the prevalence of rectification is likely to be underestimated. Specifically, when electrical synapses are identified on the basis of gap junctions seen in the electron microscope (White et al., 1986), it is unknown whether they support current flow equally in both directions or rectify. Nonetheless, in many preparations in which appropriate recordings have been made, rectifying electrical synapses have been shown to support specialized functions (Marder, 1998; Rela and Szczupak, 2004). Motor neurons in the midbody ganglion of the leech rely on rectifying gap junctions as a crucial part of a negative feedback loop built into the circuit (Rela and Szczupak, 2003). The circuit has the capacity to regulate the motor neurons in a state-dependent manner due to this circuit feature (Rela and Szczupak, 2004). The crayfish escape reflex depends on coincidence detection that results from rectifying electrical synapses. As a result of multiple inputs either summing or interfering, coincidence detection is an unavoidable feature endowed upon neurons that receive multiple rectifying electrical synapses of the same polarity (Edwards et al., 1998).

The prevalence of rectifying electrical synapses makes it important to understand the role of rectification in the circuits in which these junctions are found. To that end, we investigate how rectification affects the functional output of a 5-cell, pattern-generating, model circuit and its sensitivity to synaptic modulation.

\section{Materials and Methods}

Model networks. Cells were modeled as Morris-Lecar (Morris and Lecar, 1981), single-compartment neurons modified by a hyperpolarizationactivated current as in our previous study (Gutierrez et al., 2013). Each 

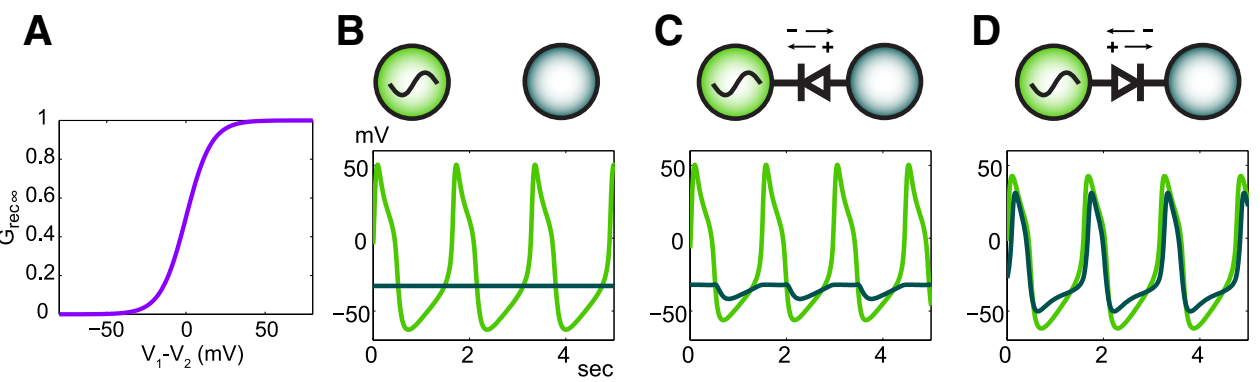

Figure 1. A rectifying gap junction between an intrinsic oscillator and a leaky oscillator. $A$, Rectification is a function of the difference in voltage between two electrically coupled cells. The rectification of the electrical synapses in this study is modeled by this $G_{\text {recos }}$ sigmoid which controls the amount of electrical coupling conductance as a function of voltage difference. $B$, The voltage traces of an intrinsically oscillating neuron (green) and a leaky, conditionally oscillating neuron (pale blue) show their intrinsic activity when they are not coupled. In $C$ they are coupled by a rectifying gap junction (diode symbol) that readily permits negative (hyperpolarizing) current to pass from the oscillator to the leaky cell while restricting the flow of negative current in the opposite direction. Naturally, positive current is able to pass from the leaky neuron into the oscillator; however, its passage into the leaky neuron from the oscillator is restricted. The voltage traces show their resulting activity. $\boldsymbol{D}$, The polarity of the rectifying gap junction is reversed so that positive current can easily pass from the oscillator to the leaky neuron and negative current can just as easily pass from the leaky neuron to the oscillator. The inverse current flow, however, is restricted.

cell's membrane voltage, $V_{m}$, was computed by solving the membrane equation:

$$
C_{m} \frac{d V_{m}}{d t}=-\left[I_{\text {leak }}+I_{c a}+I_{K}+I_{h}+I_{\text {elec }}+I_{s y n}\right] .
$$

$C_{m}$ is the membrane capacitance and is equal to $1 \mathrm{nF}$ for all neurons. Reversal potentials for the various currents are $V_{\text {leak }}=-40 \mathrm{mV}, V_{c a}=$ $100 \mathrm{mV}, V_{k}=-80 \mathrm{mV}, V_{h}=-20 \mathrm{mV}, V_{\text {syn }}=-75 \mathrm{mV}$. The hyperpolarization-activated current is based on equations and parameters that were modified from (Turrigiano et al., 1995). The remaining ionic currents are based on equations modified from (Skinner et al., 1993).

$$
I_{\text {leak }}=g_{\text {leak }}\left(V_{m}-V_{\text {leak }}\right),
$$

$I_{c a}=g_{c a} M_{\infty}\left(V_{m}-V_{c a}\right)$,

$$
M_{\infty}=0.5\left(1+\tanh \left(\frac{V_{m}-v_{1}}{v_{2}}\right)\right)
$$

where $v_{1}=0 \mathrm{mV}$ and $v_{2}=20 \mathrm{mV}$.

$$
\begin{gathered}
I_{K}=g_{K} N\left(V_{m}-V_{K}\right), \frac{d N}{d t}=\lambda_{N}\left(N_{\infty}-N\right), \\
N_{\infty}=0.5\left(1+\tanh \left(\frac{V_{m}-v_{3}}{v_{4}}\right)\right), \\
\lambda_{N}=\varphi_{N} \cosh \left(\frac{V_{m}-v_{3}}{2 v_{4}}\right),
\end{gathered}
$$

where $v_{3}=0 \mathrm{mV}$ and $v_{4}=15 \mathrm{mV}$.

$$
\begin{gathered}
I_{h}=g_{h} H\left(V_{m}-V_{h}\right), \frac{d H}{d t}=\left(\frac{H_{\infty}-H}{\tau_{h}}\right), \\
H_{\infty}=\frac{1}{1+\exp \left(\frac{V_{m}+v_{5}}{v_{6}}\right)}, \\
\tau_{h}=272-\left(\frac{-1499}{1+\exp \left(\frac{-V_{m}+v_{7}}{v_{8}}\right)}\right),
\end{gathered}
$$

where $v_{5}=78.3 \mathrm{mV}, \mathrm{v}_{6}=10.5 \mathrm{mV}, v_{7}=-42.2 \mathrm{mV}, v_{8}=87.3 \mathrm{mV}$.

$M_{\infty}, N_{\infty}$, and $H_{\infty}$ are steady-state gating variables for the calcium, potassium, and hyperpolarization-activated currents, respectively. $N$ and $H$ are time-dependent gating variables for the potassium and hyperpolarization-activated currents, respectively. The gating variable,
$N$, is modified by $\lambda_{N}$, a hyperbolic, U-shaped curve whose nadir height and eccentricity are determined by $\varphi_{N}$ which equals $0.002 \mathrm{~ms}^{-1}$. The variable $\tau_{h}$ is the voltage-dependent recovery time constant. It controls the rate of change of $H$ so that $H$ changes less steeply for more depolarized voltages.

All synapses were modeled as instantaneous. Electrical synapses were either rectifying or nonrectifying. The electrical conductance, $g_{\mathrm{e}}$, determined the strength of the electrical synapses. Electrical coupling conductances ranged from $0 \mathrm{nS}$ to as much as $9.5 \mathrm{nS}$.

$$
\begin{aligned}
I_{\text {elec }}^{\text {post }}=g_{\text {el }} G_{\text {recos }}\left(V_{m}^{\text {post }}-V_{m}^{\text {pre }}\right) & \\
G_{\text {recos }} & =G_{\min }+\frac{\left(G_{\max }-G_{m i n}\right)}{1+\exp \left(\frac{V_{m 1}-V_{m 2}}{v_{\alpha}}\right)},
\end{aligned}
$$

for a rectifying synapse that permits negative current to flow from $V_{m 1}$ to $V_{m 2}$.

The rectification function, $G_{\text {recos }}$ is an approximation of the experimental rectification data in Phelan et al. (2008). $G_{\max }=1, v_{\alpha}=8 \mathrm{mV}$, and $G_{\min }=0$ (Fig. $1 A$ ). For nonrectifying synapses, $G_{\text {recos }}$ was a constant equal to 1 .

Chemical inhibitory synapses were modeled by equations modified from Prinz et al. (2004). $S_{\infty}$ is the steady-state synaptic current gating variable and was modeled after the graded synaptic transmission seen in crustacean stomatogastric neurons (Manor et al., 1997). We used inhibitory synaptic conductance strengths $\left(g_{\text {synHC }}\right.$ and $\left.g_{\text {syn } 1}\right)$ ranging from 0 $\mathrm{nS}$ to $10 \mathrm{nS}$. Each of the two synapses that form a half-center coupling have the conductance value denoted by $g_{\text {synHC }}$.

$$
I_{s y n}=g_{s y n} S_{\infty}^{p r e}\left(V_{m}^{p o s t}-V_{s y n}\right), S_{\infty}=\frac{1}{1+\exp \left(\frac{v_{t h}-V_{m}}{v_{\beta}}\right)},
$$

where $v_{\beta}=5 \mathrm{mV}$ and $v_{\mathrm{th}}=-25 \mathrm{mV}$.

All model neurons in this study are endogenous oscillators and the maximal conductances for the different neurons were chosen to achieve the intrinsic oscillation frequencies required. Conductances for the neurons in the five-cell circuit were as follows [ fast; f1,f2: $g_{\text {ca }}=1.9 \times 10^{-2}$ $\mu \mathrm{S}, g_{k}=3.9 \times 10^{-2} \mu \mathrm{S}, g_{h}=2.5 \times 10^{-2} \mu \mathrm{S}$; hub neuron(intermediate); $h n: g_{\mathrm{ca}}=1.7 \times 10^{-2} \mu \mathrm{S}, g_{k}=1.9 \times 10^{-2} \mu \mathrm{S}, g_{h}=8.0 \times 10^{-3} \mu \mathrm{S} ;$ slow; $s 1, s 2: g_{\text {ca }}=8.5 \times 10^{-3} \mu \mathrm{S}, \mathrm{g}_{\mathrm{k}}=1.5 \times 10^{-2} \mu \mathrm{S}, g_{h}=1.0 \times 10^{-2} \mu \mathrm{S}$; all $\left.g_{\text {leak }}=1 \times 10^{-4} \mu \mathrm{S}\right]$. The neurons in Figure 1 have the same ionic conductances as $h n$ except for $g_{\text {leak }}$ which is $1 \times 10^{-3} \mu \mathrm{S}$ and $1 \times 10^{-2}$ $\mu \mathrm{S}$ for the oscillator and leaky neuron respectively. The neurons in the three-cell circuit in Figure 2 have the following conductances [f: $g_{\mathrm{ca}}=$ $2.0 \times 10^{-2} \mu \mathrm{S}, g_{k}=4.0 \times 10^{-2} \mu \mathrm{S}, g_{h}=1.9 \times 10^{-2} \mu \mathrm{S} ; \mathrm{m}: g_{\mathrm{ca}}=1.7 \times$ $10^{-2} \mu \mathrm{S}, g_{k}=2.0 \times 10^{-2} \mu \mathrm{S}, g_{h}=9.0 \times 10^{-3} \mu \mathrm{S} ; \mathrm{s}: g_{\mathrm{ca}}=1.48 \times 10^{-2}$ $\mu \mathrm{S}, g_{k}=2.5 \times 10^{-2} \mu \mathrm{S}, g_{h}=4.0 \times 10^{-4} \mu \mathrm{S}$; all $\left.g_{\text {leak }}=1 \times 10^{-4} \mu \mathrm{S}\right]$. 

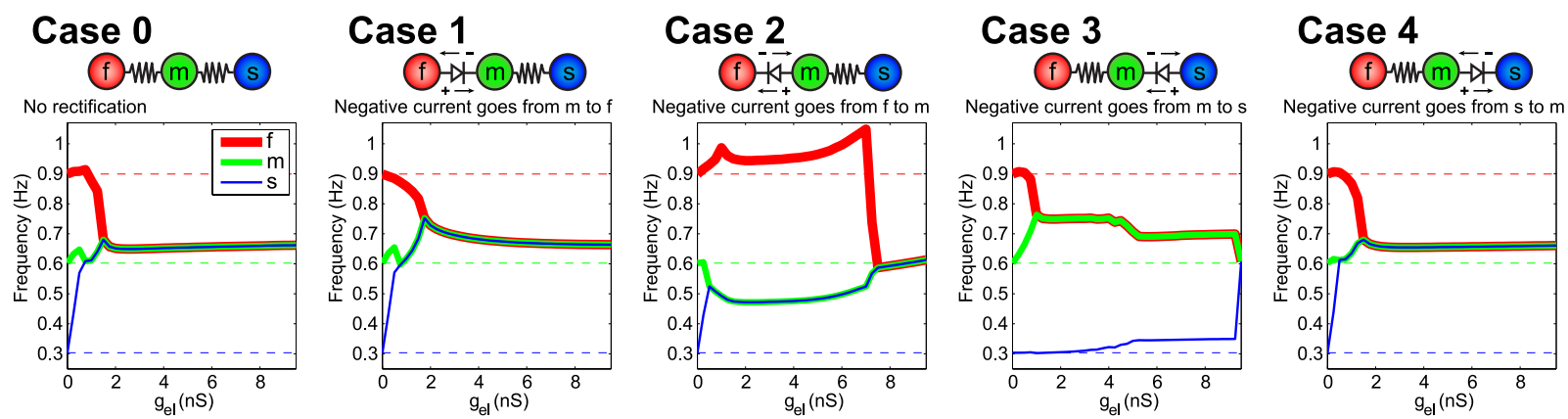

Figure 2. Synchronization properties of three heterogeneous, electrically coupled neurons. An intrinsically fast (red), medium (green), and slow (blue) oscillator (the dashed lines plotted are their respective isolated frequencies) are serially, electrically coupled with nonrectifying (Case 0 ) electrical synapses. Case 1, A rectifying synapse between the $f$ and $m$ neurons that restricts negative current from the $f$ to the $m$ neuron and a nonrectifying synapse between the $m$ and $s$ neurons. Case 2, A rectifying synapse between the $f$ and $m$ neurons that restricts negative current from the $m$ to the $f$ neuron and a nonrectifying synapse between the $m$ and $s$ neurons. Case 3, a rectifying synapse between the $m$ and $s$ neurons that restricts negative current from the $s$ to the $m$ neuron and a nonrectifying synapse between the $m$ and $f$ neurons. Case 4 , a rectifying synapse between the $m$ and $s$ neurons that restricts negative current from the $m$ to the $s$ neuron and a nonrectifying synapse between the $m$ and fneurons. Neuron oscillation frequencies are plotted as a function of electrical synapse conductance in nanosiemens (nS). Both synapses in the circuit are varied together along the $x$-axis. Resistor symbols are nonrectifying electrical synapses and diode symbols are rectifying electrical synapses.

Simulations and analysis. All data and figures presented in this paper are from simulations performed in MATLAB using the variable timestep solver ode 45 function (fourth/fifth order Runge-Kutta integration). Simulations produced $655 \mathrm{~s}$ long voltage waveforms for each of the five neurons. The first $55 \mathrm{~s}$ were eliminated from analysis. MATLAB scripts were made to compute the oscillation frequencies from the truncated 600 s lengths of data. The threshold for a membrane depolarization to be considered a spike was set at $0 \mathrm{mV}$ and frequency was calculated to be the inverse of the mean spike period over the $600 \mathrm{~s}$ interval. Network parameterscapes were done in MATLAB as described in Gutierrez et al. (2013). Code available on request.

\section{Results}

Many computational studies of the effects of gap junctions in networks assume that all of the component neurons are identical and have not investigated the effects of rectification. In contrast, in biological systems, many electrical synapses are between neurons of different cell types, and may be rectifying. Therefore, we focus on the effects of rectifying junctions between neurons with different intrinsic membrane properties. We begin with an example of two electrically coupled neurons because it offers some insights into the findings that follow when we look at a chain of three heterogeneous oscillators. The three-cell chain then forms the kernel of the five-cell pattern-generating circuit that we use to explore the dynamics that result from rectification in a network with both electrical and chemical synapses.

\section{Rectification between two heterogeneous neurons}

The effect of a rectifying electrical synapse between an intrinsically oscillating neuron and a leaky neuron is shown in Figure 1. Uncoupled, the oscillator's membrane potential fluctuates between depolarized and hyperpolarized voltages, whereas the leaky neuron's membrane potential is steady at around the leak reversal potential (Fig. 1B). With a rectifying electrical synapse that restricts the flow of negative current from the leaky neuron to the intrinsic oscillator, whereas positive current flow is restricted in the opposite direction, the leaky neuron is hyperpolarized during the trough of the oscillator's waveform and the intrinsic oscillator's frequency is slightly increased (Fig. 1C). Reversing the polarity of the rectifying electrical synapse restricts negative current flowing from the intrinsic oscillator to the leaky neuron. With this configuration, both neurons oscillate synchronously (Fig. 1D). Although in Figure $1 C$ the leaky neuron only really hyperpolarizes relative to its baseline, in Figure $1 D$ the leaky neu- ron both depolarizes and hyperpolarizes relative to its baseline. The negative deflections in the leaky neuron's membrane potential in Figure $1 D$ are due to the activation of its intrinsic hyperpolarizing membrane currents as a result of its depolarization through the rectifying gap junction. Figure 1 illustrates that, depending on the direction of rectification, the oscillatory neuron can either produce a net rhythmic hyperpolarization or a net rhythmic depolarization to the leaky coupled neuron. More importantly, the intrinsic properties of the neurons determine how the rectifying electrical synapse will affect their activity as demonstrated by Figure $1 D$. Although this is to be expected for this pair, it is important to bear in mind for the larger circuits we study subsequently.

The current flow and its rectification are a function of the voltage difference between the coupled neurons (Fig. 1A). Take for example the rectifying synapse in Figure $1 C$; when the oscillator is more hyperpolarized than the leaky neuron, $V_{\text {leaky }}-V_{\text {oscillator }}$ is positive and $G_{\text {reco }}$ is between 0.5 and 1 meaning that current flow through the gap junction is facilitated. When the oscillator is more depolarized than the leaky neuron, $V_{\text {leaky }}-V_{\text {oscillator }}$ is negative and $G_{\text {recos }}$ is between 0 and 0.5 which means that current flow through the gap junction is restricted. Note how the more depolarized the oscillator is than the leaky neuron, the more current flow is restricted. For the rectifying gap junction of the reverse polarity in Figure $1 D$, the $G_{\text {recos }}$ sigmoid is reflected about the axis where $V_{1}-V_{2}=0$.

\section{Rectification in a three-cell chain of heterogeneous neurons}

We next look at the effects of rectification on a three-cell circuit that demonstrates that the effect that a rectifying electrical synapse has on circuit synchronization depends on the polarity of the synapse and the intrinsic properties of the neurons on either side of it.

In isolation, each of the three neurons in Figure 2 is an oscillator, but their frequencies are different. If the neurons are serially coupled with nonrectifying electrical synapses, they oscillate synchronously near the mean of their isolated frequencies given enough electrical coupling conductance (Fig. 2, Case 0). In this case, the slow $(s)$ and the medium $(m)$ frequency neurons synchronize at a slightly lower value of the coupling conductance than that required before all three synchronize. Replacing the electrical synapse between the intrinsically fast $(f)$ and mediumfrequency neurons with a rectifying electrical synapse that restricts negative current from the fast to the medium neuron (and 
restricts positive current in the opposite direction) yields similar results, but shifts the coupling conductance needed to synchronize the three neurons to a slightly higher value (Fig. 2, Case 1). However, a rectifying synapse of the opposite polarity between the fast and medium neurons results in a circuit that requires a much higher coupling conductance before all three neurons synchronize (Fig. 2, Case 2).

The contrast between these two cases has an intuitive explanation. Because in isolation the fast neuron oscillates at a higher frequency than the other two neurons, the hyperpolarizing current it receives from the medium neuron when they are coupled with the rectifying synapse in Case 1 allows it to lower its oscillation frequency to meet the other neurons. When the polarity of the rectifying synapse is reversed, the fast neuron's source of hyperpolarizing synaptic current is restricted, whereas the depolarizing current it receives pushes its frequency further away from that of the other neurons until a high enough coupling conductance can synchronize them. As coupling conductance is increased, the fast neuron is able to receive more negative synaptic current when the junctional potential is near zero. Similarly, Cases 3 and 4 (Fig. 2) show how the polarity of a rectifying synapse can work for, or against, the intrinsic properties of the neurons flanking it and this can have consequences for circuit output. In Case 3, restricting positive current to the slow neuron makes it difficult for it to synchronize with the other faster neurons. However, in Case 4, the slow neuron is able to synchronize with the other neurons at a lower coupling conductance despite having negative current flow into the slow neuron restricted. The rectifying electrical synapses in Cases 1 and 4 cooperate with the neuron intrinsic properties because the sign of the permitted current flow through the synapse facilitates synchrony among the coupled neurons. Hyperpolarizing current flows easily from $m$ to $f$ in Case 1 , whereas in Case 4 depolarizing current flows easily from $m$ to $s$, allowing $f$ to decrease its frequency and $s$ to increase its frequency to meet the other neurons in the respective cases. Conversely, in Cases 2 and 3 the relationship is antagonistic because the asymmetrical current flow through the rectifying electrical synapse results in the coupled neurons diverging in frequency. More explicitly, in Case 2 depolarizing current flows easily from $m$ to $f$ causing the frequency of $f$ to increase away from the other neurons. In Case 3, because hyperpolarizing current flows more easily from $m$ to $s$, the slow neuron's frequency is suppressed making it difficult for $\mathbf{s}$ to synchronize with the other neurons.

Consequences of rectification for a pattern-generating circuit In the three-cell circuit, we saw the effects of rectification on synchronization among cells of disparate frequencies. Now, we look at the effects of rectification in a circuit that can generate a variety of different output patterns, such as in the central pattern generating circuits seen in invertebrates (Marder and Calabrese, 1996; Marder and Bucher, 2007). As an example, we study the effects of rectification on a five-cell circuit that is loosely motivated by the connectivity in the crab stomatogastric ganglion (Gutierrez et al., 2013). In this circuit there are two intrinsically fast neurons ( $f 1$ and $f 2$ ) that mutually inhibit each other, two intrinsically slow neurons ( $s 1$ and $s 2$ ) that mutually inhibit each other, and an intermediate-frequency hub neuron $(h n)$ that is electrically coupled to one of the fast and one of the slow oscillators (Fig. 3A).

Both mutually inhibitory pairs (coupled with synaptic strength $g_{\text {synHC }}$ ) form half-center oscillators as seen in the traces in Figure $3 B$. This endows the circuit with pattern-generating

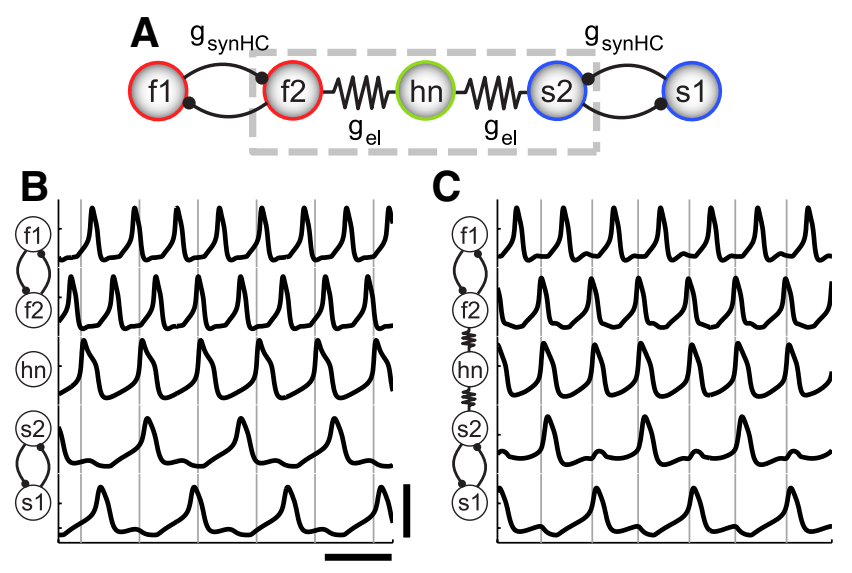

Figure 3. Pattern-generating five-cell circuit. $\boldsymbol{A}$, The electrically coupled three-cell circuit motif becomes the core of the five-cell circuit. $\boldsymbol{B}$, The intrinsically fast neuron is mutually inhibited by another intrinsically fast neuron forming a half-center oscillator, and likewise for the slow neuron ( $g_{\text {synHc }}=5 \mathrm{nS}$ ). Without electrical coupling, the hub neuron $(h n)$ between these two pattern-generating components intrinsically oscillates at a frequency intermediate to the half-center frequencies of the fast and slow pair. $\boldsymbol{C}$, When $h n$ is electrically coupled to $f 2$ and $s 2$ with $\mathrm{g}_{\mathrm{el}}$ equal to $3 \mathrm{nS}$, the circuit outputs a stable rhythmic pattern and $h n$ oscillates at the frequency of the fast half-center. Scale bars, $100 \mathrm{mV}$ and $2 \mathrm{~s}$.

capabilities such that the mutually inhibitory pairs set up two distinct rhythmic patterns. In the half-center oscillator formed by the two fast neurons, $f 1$ and $f 2$ take turns "firing" in alternation. The slow neurons also form a half-center oscillator but at a lower frequency than the fast half-center. The hub neuron, $h n$, between the rhythm-generating parts of the circuit intrinsically oscillates at a frequency intermediate to the fast and slow half-center frequencies (Fig. $3 B$ ). In contrast to the three-cell circuit, $h n$ is flanked by two half-center subcircuits of different frequencies in this five-cell circuit, rather than by two neurons with differing frequencies. The electrically coupled neurons on either side of $h n$ now have the additional pull of their mutually inhibiting partners to compete with the influence of the electrical coupling.

The fast and slow rhythms can be coordinated by $h n$ via the electrical coupling (Fig. $3 C$ ). When $h n$ is electrically coupled to one neuron in each half-center oscillator ( $f 2$ and $s 2$ ), it can coordinate the two sides of the circuit such that the fast and slow half-centers fire in a stable 2:1 frequency relationship with each other. In the example in Figure $3 C, h n$ joins the fast half-center oscillator's frequency.

In this five-cell circuit, electrical coupling competes with the half-center coupling (i.e., the mutually inhibitory chemical synapses) to determine the electrically coupled cells' activity. With low half-center inhibitory coupling conductance, the electrically coupled neurons ( $f 2, h n$, and s2) synchronize at the same frequency while the nonelectrically coupled neurons ( $f 1$ and $s 1$ ) oscillate close to their intrinsic frequencies (Fig. 4A). However, the effect of these synaptic parameters is not limited to the activity of the electrically coupled neurons, but extends to the nonelectrically coupled ones as well. This is seen in Figure $4 B$ in which, as a result of increasing both electrical and half-center coupling strength, the nonelectrically coupled intrinsically fast neuron ( $f 1$ ) has joined the electrically coupled neurons at the same frequency leaving only the nonelectrically coupled, intrinsically slow neuron $(s 1)$ to oscillate near its intrinsic frequency. Naturally, high half-center coupling conductance will make it less likely for the neurons on either side of the hub neuron to be 
A

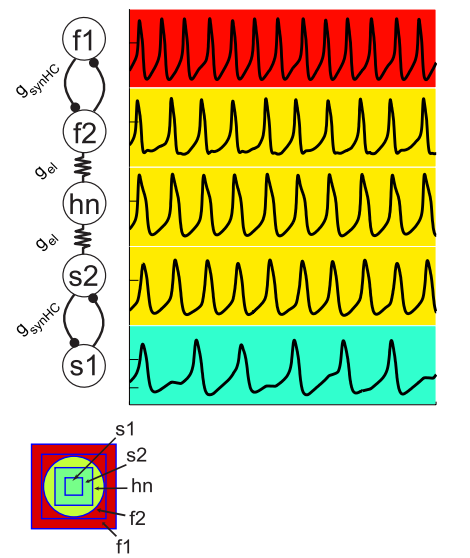

B

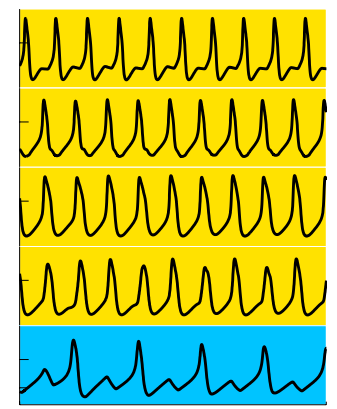

Neuronal Circuit Frequencies

C

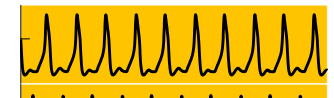

musult
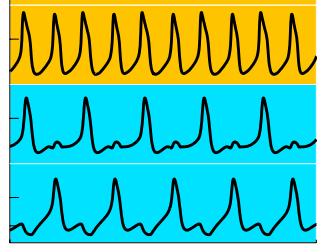

Case 0: No rectification
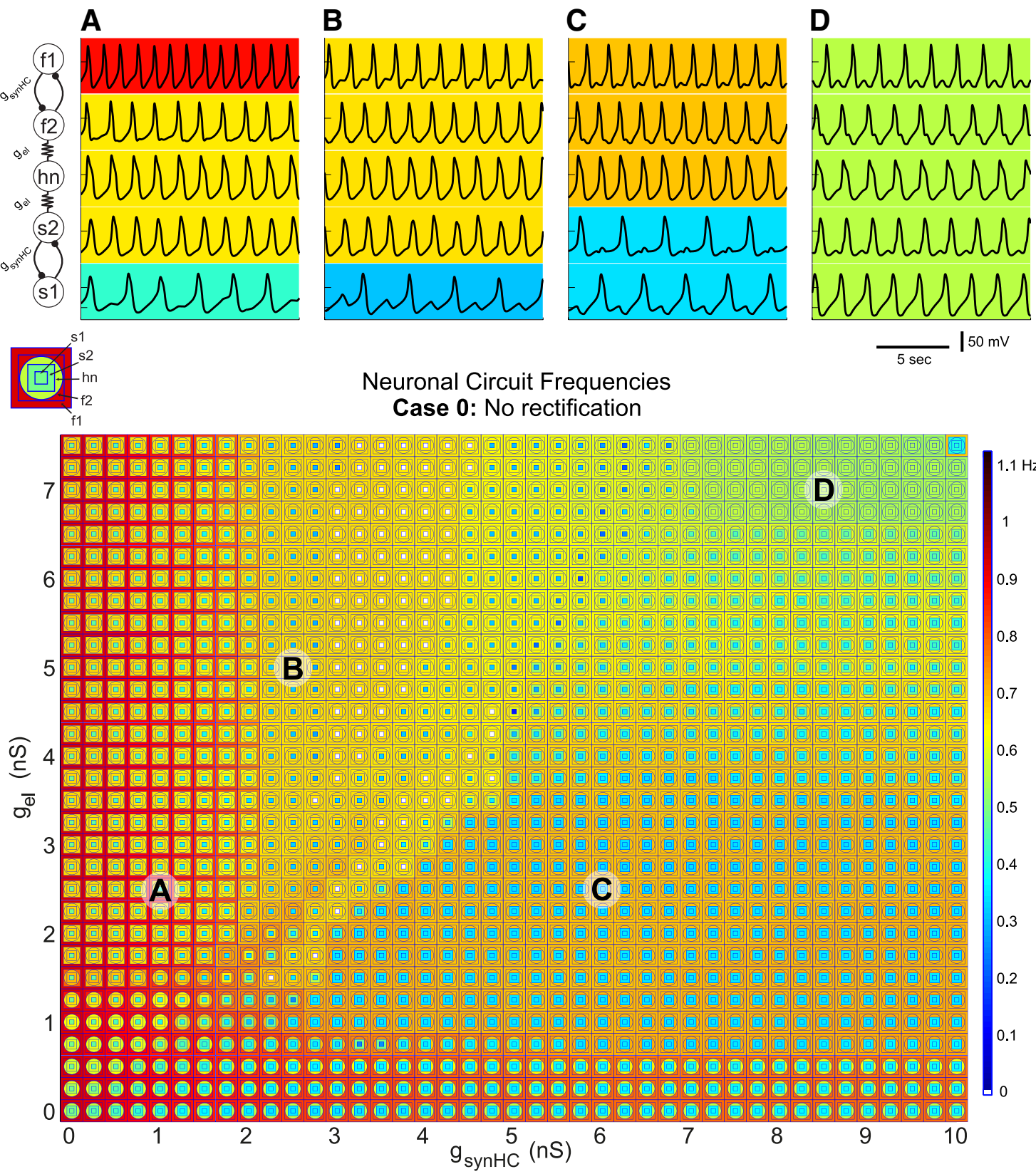

Figure 4. Circuit output as a function of $g_{\text {synHc }}$ and $g_{\mathrm{el}} \cdot \boldsymbol{A}-\mathbf{D}$, Voltage traces show the activity of the circuit with nonrectifying electrical synapses for various conductance strength combinations. $A, g_{\text {synHC }}=1 \mathrm{nS}, g_{\mathrm{el}}=2.5 \mathrm{nS} ;(\boldsymbol{B}) g_{\text {synHC }}=2.5 \mathrm{nS}, g_{\mathrm{el}}=5 \mathrm{nS},(\boldsymbol{C}) g_{\text {synHC }}=6 \mathrm{nS}, g_{\mathrm{el}}=2.5 \mathrm{nS}$; and (D) $g_{\text {synHC }}=8.5 \mathrm{nS}, g_{\mathrm{el}}=7 \mathrm{nS}$. Background colors indicate neuron oscillation frequency. Parameterscape (below) shows circuit activity for a range of electrical $\left(g_{\mathrm{el}}\right)$ and half-center coupling ( $\left.g_{\text {synht }}\right)$ conductance combinations with overlapping concentric shapes at each point. Key (above left of parameterscape) shows which neuron corresponds to each concentric shape. Colors represent suprathreshold voltage oscillation frequency (color axis) in hertz with blue shades indicating low frequencies and red shades indicating high frequencies.

pulled out of synchrony with their mutually inhibiting partners, so $h n$ will join one of the half-center oscillators. In Figure $4 C$, the half-centers ( $f 1, f 2$, and $s 1, s 2)$ oscillate near their intrinsic frequencies, whereas $h n$ is captured by the fast oscillators. When both half-center coupling and electrical coupling are high, the network finds a stable compromise between the opposing forces such that half-center relationships are preserved while the three electrically coupled neurons synchronize. For example, in Figure $4 D$, all neurons oscillate at a common frequency, but alternation between $f 1$ and $f 2$, and between $s 1$ and $s 2$ is maintained.

The addition of the half-center coupling parameter requires a plotting method to accommodate this added dimension when probing a range of synaptic conductances. We recently developed a method called the "parameterscape" that allows the visualization of any network attribute (in this case the frequencies of the five circuit neurons) as a function of two network parameters (Gutierrez et al., 2013). Each point on the parameterscape shows the activity of all five neurons with a series of concentric shapes that are color-coded for oscillation frequency (Fig. 4, parameterscape key). At each point, the circuit activity for a particular set of synaptic conductances can be ascertained at a glance. The patterns of network activity shown in the traces of Figure 4 can be seen at the points labeled $A-D$ in the parameterscape below the traces. The electrically coupled neurons ( $f 2, h n$, and s2) synchronize whenever $g_{\text {el }}$ is greater than $g_{\text {synHC }}($ and $>1.5 \mathrm{nS}$ ), but this relationship is not linear. 
We hypothesized that the network output patterns generated by this five-cell circuit would be changed by making any of the electrical synapses rectifying. So we generated parameterscapes for four cases of rectification. The rectification configurations from Figure 2 are used for the three electrically coupled neurons within the five-cell circuit. Recall that in Case 1, the electrical coupling between $f 2$ and $h n$ is rectifying so that negative current flows freely from $h n$ to $f 2$. The polarity of this rectifying synapse between $f 2$ and $h n$ is reversed in Case 2. Case 3 has a rectifying electrical synapse between $s 2$ and $h n$ such that negative current flows freely from $h n$ to $s 2$ and Case 4 has the opposite polarity synapse. Indeed, the effect of rectification on network output in the five-cell circuit echoes that in the three-cell circuit. For Cases 1 and 4 where the rectifying electrical synapses cooperate with the intrinsic properties of the neurons, the parameterscapes (data not shown) looked similar to the parameterscape in Figure 4 in which none of the synapses are rectifying. However, for Cases 2 and 3 in which the rectifying electrical synapses have an antagonistic relationship with the neurons' intrinsic properties, the parameterscapes were drastically changed from that seen in Figure 4 . The parameterscape for Case 2 (Fig. 5, top) is dominated by network behavior in which $h n$ oscillates with the intrinsically slow halfcenter pair leaving the intrinsically fast half-center oscillator active at a higher frequency. However, in Case 3 (Fig. 5, bottom) $h n$ oscillates with the intrinsically fast half-center oscillators for most of the synaptic conductance strengths in the parameterscape. In each of these two cases, the rectifying electrical synapse effectively gates off one of the rhythm-generating parts of the circuit leaving it to be active on its own, whereas the nonrectifying synapse facilitates synchrony between $h n$ and the other half-center pair. This effective "gating-off" leads to robustness of one network pattern within the parameterscape and makes other network patterns "forbidden," at least in the synaptic conductance ranges studied (simulations were run for $g_{\text {el }}$ values up to $9.5 \mathrm{nS}$, but as conclusions were not affected by the extended range, only values up to $7.5 \mathrm{nS}$ are plotted here).

An intuition about the gating-off effect discussed above can be gained from the three-cell circuit in Figure 2. In fact, by choosing a single half-center conductance value and focusing on a column of a parameterscape in Figure 5, the frequencies of the electrically coupled neurons as $g_{\text {el }}$ is increased follow a similar pattern in the five-cell circuit as in the three-cell circuit. The only difference is that a point at which all three electrically coupled neurons in the five-cell circuit synchronize is never reached in Cases 2 and 3 of Figure 5.

\section{Rectification and parallel pathways}

Many neuronal circuits have parallel pathways between neurons that impact the complexity and robustness of the circuit. In this context, parallel pathways enable one neuron to communicate with another neuron via multiple routes, and can include both monosynaptic and polysynaptic connections. Parallel pathways can permit the nature of the communication to differ depending on modulatory state. For example, a neuron that sends both an inhibitory chemical synapse and an electrical synapse to its postsynaptic partner can have either a desynchronizing or synchronizing effect depending on the strengths of these synapses (Lewis and Rinzel, 2003; Bem and Rinzel, 2004). An intuition about the gating-off effect in Figure 5 was gained from studying the threecell circuit, but how might this gating be affected if there are parallel pathways through which the hub neuron can be dually influenced by the pattern-generating components of the circuit? To address this question, we added inhibitory synapses from the nonelectrically coupled neurons to $h n$. The behavior of this particular circuit with only nonrectifying synapses was studied in detail in Gutierrez et al. (2013). Here, we focus on the impact of rectification on this circuit and develop a comparison between the network outputs in Cases 2 and 3 with and without parallel pathways.

The conductances of the synapses that form the half-centers were set to a constant value of $5 \mathrm{nS}$, whereas the conductances of the inhibitory synapses onto $h n$ were varied, as well as the electrical coupling conductances. In the parameterscapes in Figure 6, the conductances of the inhibitory synapses that project to $h n$ ( $\left.g_{\text {syn } 1}\right)$ are varied along the $x$-axis.

In relating the parameterscapes in Figure 5 to those in Figure 6 , it is helpful to note that the first columns of the parameterscapes in Figure 6 correspond to the middle columns in the parameterscapes in Figure 5, where $g_{\text {synHC }}$ is $5 \mathrm{nS}$. Modulation of only $g_{\text {el }}$ and $g_{\text {syn } 1}$ results in homogenous network behavior in Case 2 (Fig. 6, top). That means that the network only produces one pattern for this range of synaptic conductances. Regardless of whether $g_{\text {syn } 1}$ is much higher or lower than $g_{\text {el }}, h n$ is always active with the slow rhythm except when both $g_{\text {syn } 1}$ and $g_{\text {el }}$ are very low $(<1 \mathrm{nS})$. The striking homogeneity of this parameterscape reveals this network's insensitivity to the modulation of not only electrical synapse conductance but also the modulation of the inhibitory synapses impinging onto $h n$.

In Case 3, the parallel pathways have the inverse effect by expanding the repertoire of circuit behaviors (Fig. 6, bottom). In other words, the circuit for Case 3 in Figure 6 displays more than one output pattern as a function of the electrical and parallel inhibitory synapse strengths in contrast to Case 2 and, more importantly, in contrast to Case 3 in Figure 5. The inhibitory synapse from $s 1$ to $h n$, in effect, compensates for the rectification of the electrical synapse between $s 2$ and $h n$ because it provides some of the slow, rhythmic hyperpolarization that the rectifying electrical synapse restricts. Thus, $h n$ can be recruited into the slow rhythm for high enough $g_{\text {syn } 1}$ values despite the antagonistic relationship between the rectifying electrical synapse and the neurons' intrinsic properties. Furthermore, at the highest values of $g_{\text {syn } 1}$ and $g_{\text {el }}$ (Fig. 6, bottom; top right part of the plot), $f 2$ can also be recruited into the slow rhythm leaving $f 1$ to oscillate at a higher frequency alone. Despite their disparate frequencies, $f 1$ and $f 2$ maintain their half-center relationship by oscillating in a $2: 1$ frequency pattern. For the lower range of $g_{\text {syn } 1}$ values, the circuit displays the same robust pattern that dominated the parameterscape in Case 3 of Figure 5. In Case 3 of Figure 6, however, the parallel pathway behaves like a switch that determines whether or not modulation of electrical synapse conductance will be able to modify the circuit pattern. When $g_{\text {syn } 1}$ is low, the circuit is robust against modulation of $g_{\mathrm{el}}$ but when $g_{\text {syn } 1}$ is high, modulation of $g_{\mathrm{el}}$ allows the circuit to switch between stable activity patterns.

The main difference between the two cases in Figure 6 is that $g_{\text {syn } 1}$ compensates for the rectification in Case 3, whereas it reinforces the effect of rectification in Case 2. This difference is due to the differing intrinsic oscillatory drives among the neurons in the circuit and the effect that the inhibitory synapses have on $h n$. The addition of the parallel pathways to this circuit shows how rectification of an electrical synapse has the potential to add complexity to a neuronal circuit on its own and to completely change the circuit's function.

\section{Discussion}

Although it is now clear that electrical synapses are ubiquitously found in all nervous systems, their roles in circuit dynamics and 


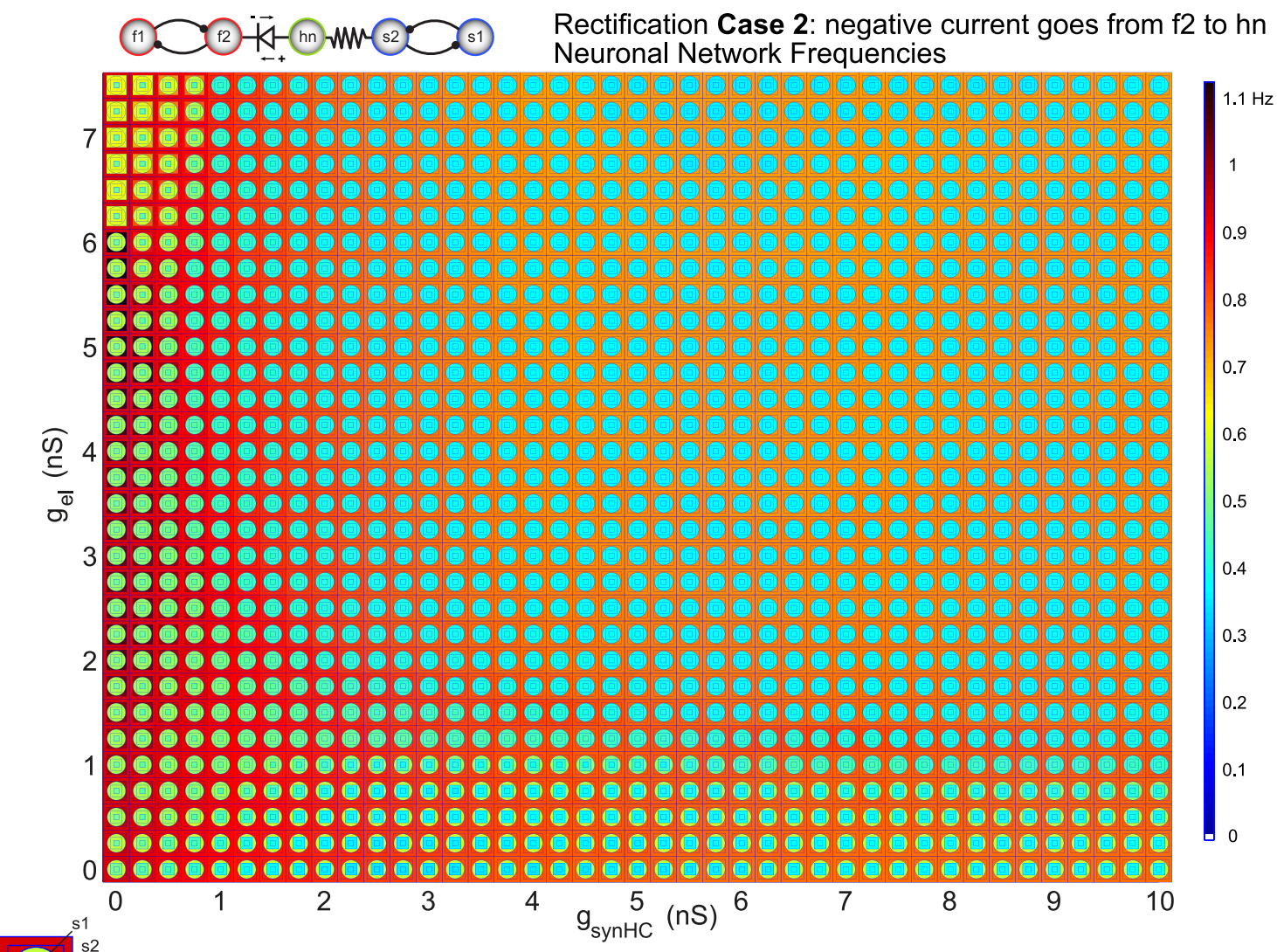

Rectification Case 2: negative current goes from f2 to hn Neuronal Network Frequencies

(b) - ${ }^{-1 n}$ Rectification Case 3: negative current goes from hn to s2 f1

Neuronal Network Frequencies

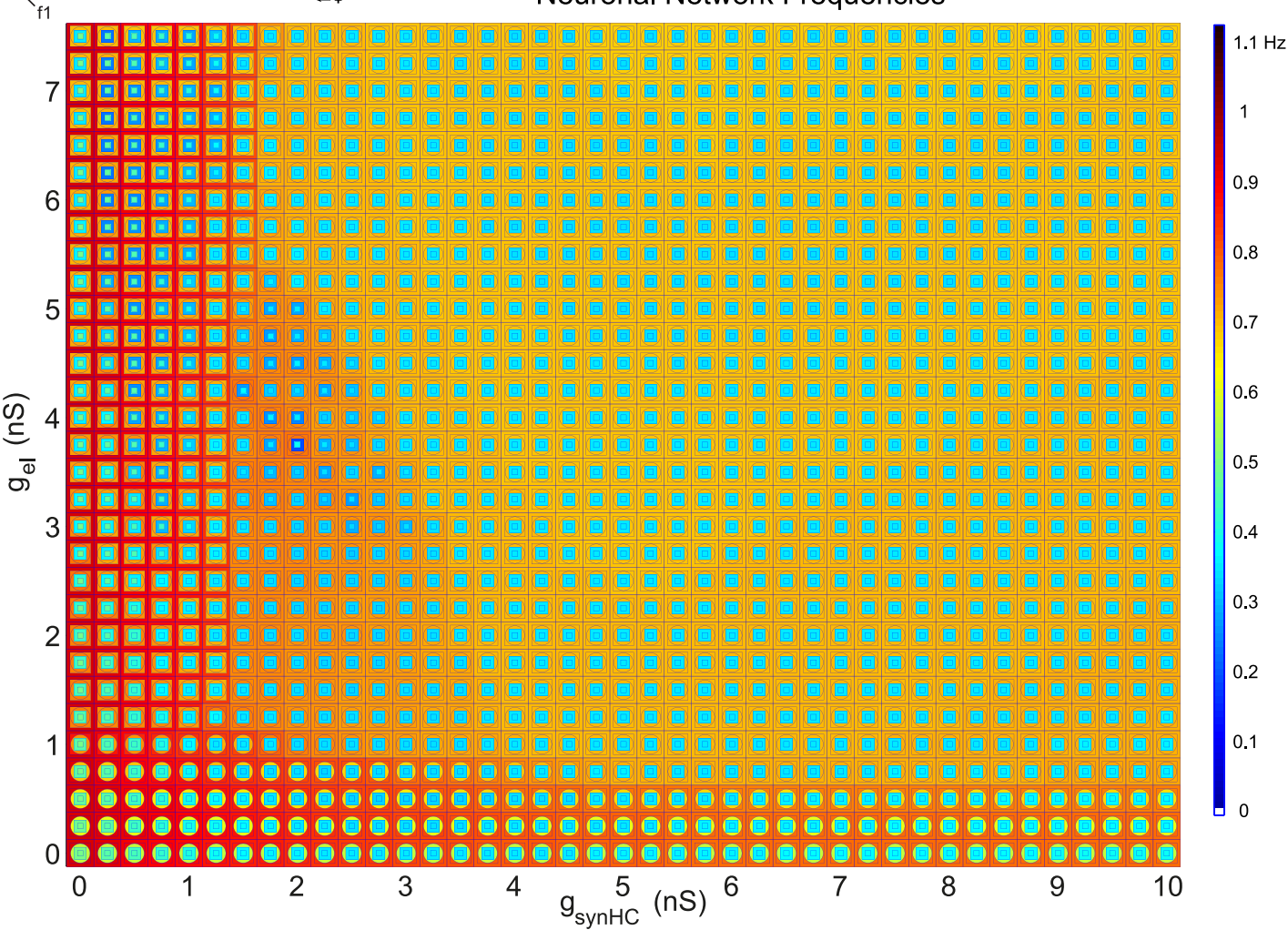

Figure 5. Circuit output with rectification. Parameterscapes of neuron frequencies for Case 2 rectification (top) and Case 3 rectification (bottom) in the five-cell circuit. The half-center coupling conductance is varied along the $x$-axis $\left(g_{\text {synHc }}\right)$, while electrical coupling conductance is varied along the $y$-axis $\left(g_{\mathrm{el}}\right)$ as in the parameterscape in Figure 4. 


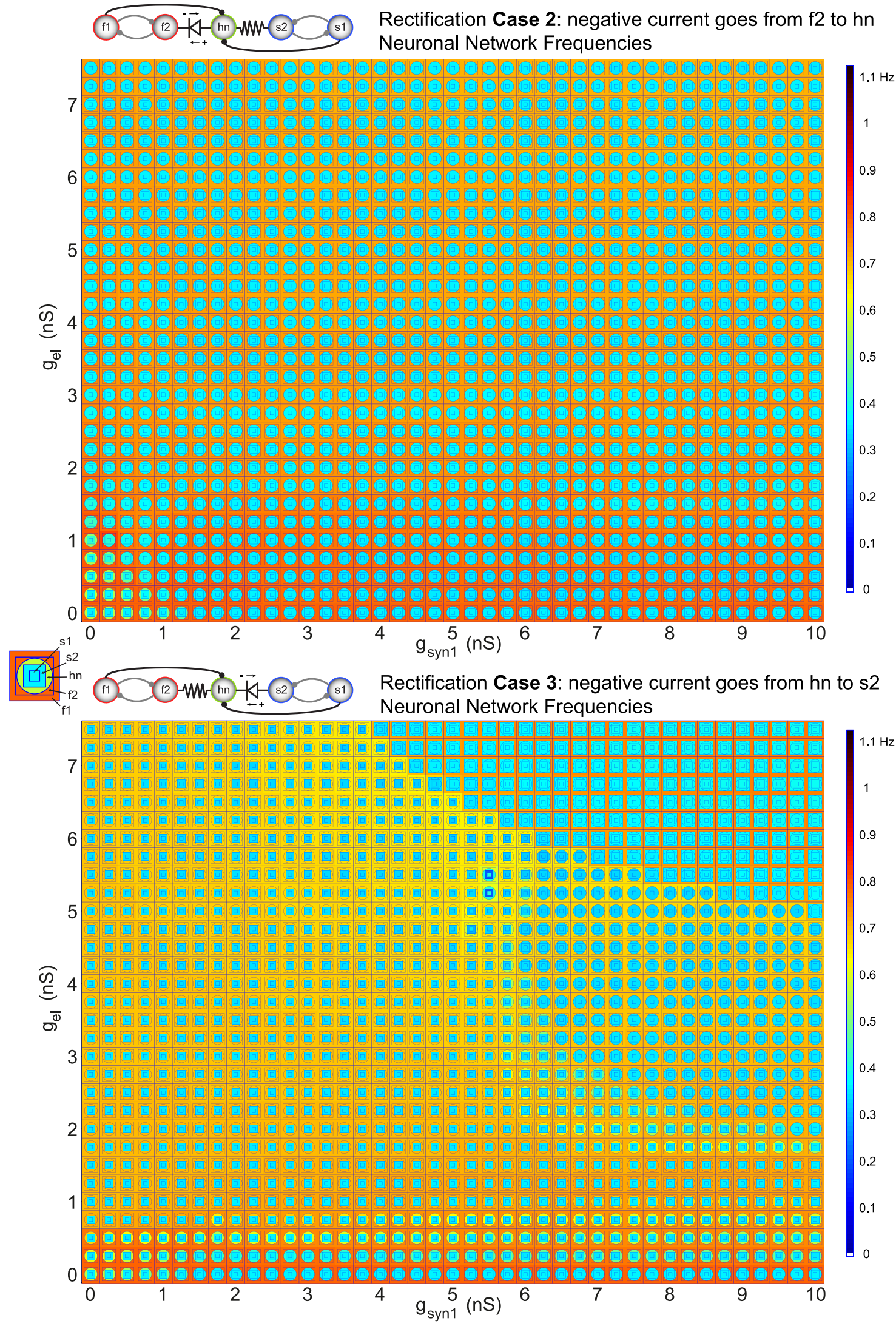

Figure 6. Circuit output with rectification and parallel pathways. Parameterscapes of neuron frequencies for Case 2 rectification (top) and Case 3 rectification (bottom) in the five-cell circuit with parallel pathways to $\mathrm{hn}$. Half-center coupling $\left(g_{\text {synHc }}\right)$ is kept constant at $5 \mathrm{nS}$ throughout the parameterscapes. The conductances of the inhibitory synapses from $s 7$ and $f 1$ onto $h n$ are varied along the $x$-axis $\left(g_{\text {syn1 }}\right)$, whereas electrical coupling conductance is varied along the $y$-axis $\left(g_{\mathrm{el}}\right)$. 
behavior have seen less computational study than chemical synapses. This relative neglect is even more so for rectifying electrical synapses, which have been largely ignored computationally, although they are commonly found in animals of disparate phyla (Furshpan and Potter, 1959; Johnson et al., 1993; Edwards et al., 1998; Phelan et al., 2008). Because nonrectifying junctions are bidirectional, but rectifying junctions pass current preferentially in one direction, the extent of rectification may significantly alter the role of gap junctions in circuit performance. This is particularly important for interpreting the results of connectome studies that derive from electron microscopic methods (White et al., 1986; Chklovskii et al., 2010; Varshney et al., 2011). Indeed, the prevalence of rectification is probably underestimated because it requires electrophysiological measurements from coupled cells to demonstrate that the rectification exists. Therefore, many of the efforts to go from an anatomically derived connectome to understanding function may be disappointing as a consequence of the presence of both rectifying electrical junctions and parallel pathways.

\section{Rectification changes sensitivity to synaptic strength}

Past work has shown that electrical synapses even without rectification can have counterintuitive and complex effects (Kopell et al., 1998; Bem and Rinzel, 2004). An early model of electrically coupled Inferior Olive neurons demonstrated that electrical coupling can produce four modes of synchronization among neuron pairs, including in-phase and anti-phase synchrony, depending on modulation of the ionic conductances and coupling strengths (Schweighofer et al., 1999). Furthermore, membrane conductances are able to regulate the efficacy of electrical synapses thus providing a mechanism to amplify input from electrically coupled neurons (Curti and Pereda, 2004; Dugué et al., 2009). This has been shown to be an important mechanism in the synchronization of neuron populations in the mesencephalic trigeminal nucleus of rats and mice (Curti et al., 2012).

One of the most general findings of this work is that rectification can completely transform the extent to which the output of a circuit depends on the strengths of its chemical and electrical synapses. This is seen most dramatically by comparing the parameterscape in Figure 4 with those in Figure 5. In Figure 4, without rectification, the parameterscape shows a variety of network behaviors as the strengths of the chemical and electrical synapses are varied. Remarkably, in Figure 5 we see the same general network architecture but now for two of the cases of rectification, the network output is substantially less affected by changing the strengths of the electrical and mutually inhibitory chemical synapses. This is even more dramatic for the network shown in Figure 6, Case 2. Here, the rectification makes the behavior of the network almost completely insensitive to the strengths of the electrical and parallel chemical synapses, although without rectification the network shows a full range of output patterns (Gutierrez et al., 2013). Why does this occur? In Case 2 for the five-cell circuit, depolarizing current flow is limited from $f 2$ to $h n$. This makes it difficult for $h n$ to increase its frequency to oscillate with the fast rhythm. In Case 3, hyperpolarizing current from $s 2$ to $h n$ is limited. This means that the hyperpolarizing drive from the slow neurons to decrease $h n$ 's frequency must rely more on the synaptic inhibition from $s 1$ than on the hyperpolarizing current from the rectifying synapse. This explains why the parameterscape in Figure 6, Case 3, displays more network patterns than Case 3 in Figure 5. It also provides an example of a parallel pathway that compensates for a rectifying electrical synapse.
Thus, the inclusion or exclusion of rectifying electrical synapses in a neuronal circuit may determine how robust the circuit output is to modulation of other synaptic strengths. This also illustrates directly the limitations of a connectome without knowledge of the properties of the underlying junctions.

\section{Biological interpretation}

The rectification function used in this study was based on an approximation of the gap junction rectification measured between Xenopus oocytes with heterotypic gap junction channels (Phelan et al., 2008). However, rectification functions differ widely across preparations (Suchyna et al., 1999; Evans and Martin, 2002), and the degree of rectification and the steepness will depend on the hemi-channel combination (White et al., 1995). Because a vast number of gap junction hemi-channel combinations are possible, this potentially could lead to a significant diversity of possible rectification properties, even within the same circuit under different conditions. Therefore, this study was not intended to model the degree of rectification of a specific form of gap junction, but to establish a set of potential consequences that rectification could have for circuit function.

Although we modeled rectifying electrical synapses explicitly, apparent electrical synapse rectification has also been found between neurons with different intrinsic properties (Nolan et al., 1999; Rela and Szczupak, 2004). Symmetrical junctional potentials were measured between electrically coupled AII neurons in the retina, indicating that the gap junctions were not rectifying, yet the coupling coefficients were asymmetrical. This apparent rectification was found to be a result of the differing input resistances between the neurons (Veruki and Hartveit, 2002). Neuronal circuits with electrically coupled heterogeneous neurons may contend with functional rectifying electrical transmission whether or not their gap junctions are themselves rectifying.

\section{Modulation of rectification could alter the function of a circuit}

It is known that neuromodulatory substances can alter the strength of gap junctions (Piccolino et al., 1982, 1984; Neyton and Trautmann, 1985, 1986a,b; Johnson et al., 1993; McMahon and Brown, 1994; Bloomfield and Volgyi, 2009) and influence circuit output (Jang et al., 2012). For example, in the retina, dopaminergic modulation of gap junctions affects processing of light stimuli (Bloomfield and Volgyi, 2009), and a variety of hormones and neurotransmitters influence coupling in the uterus and elsewhere (Neyton and Trautmann, 1986a). Gap junction proteins are often the targets of phosphorylation, and cAMP and CaMKinase II are known to regulate some connexons (Hormuzdi et al., 2004; Söhl et al., 2005; Bloomfield and Volgyi, 2009). Furthermore, gap junctions are subject to activity-dependent modulation (Haas et al., 2011; Haas and Landisman, 2011). Therefore, we speculate that the extent to which a given electrical synapse shows rectification may also be subject to modulation, either by activity that produces long-term inactivation of a conductance, or directly by neuromodulatory substances. The results of this paper demonstrate that if the extent of rectification of a given electrical synapse were to be modulated, this could dramatically alter the output of the circuit, and its robustness to changes in other parameters.

Many of the known examples of rectification are found in well defined invertebrate sensory-motor circuits (Furshpan and Potter, 1959; Muller and Scott, 1981; Johnson et al., 1993; Edwards et al., 1998; Phelan et al., 2008). In general, invertebrate central 
pattern generating circuits are highly modulated (HarrisWarrick and Marder, 1991; Marder, 2012), and these circuits are often multifunctional, with neurons that can combine into different circuit ensembles under modulatory control (Hooper and Moulins, 1989; Dickinson et al., 1990; Weimann and Marder, 1994; Briggman and Kristan, 2008; Friedman et al., 2009; Beverly et al., 2011; Jang et al., 2012; Sasaki et al., 2013). Electrical synapses are common in these networks, and undoubtedly play important roles in the network reconfigurations that underlie these changes in behavior. We now suggest that modulation of the extent of rectification of one or more of the electrical synapses in a network could be a novel mechanism underlying circuit reconfiguration in behavior.

\section{References}

Bem T, Rinzel J (2004) Short duty cycle destabilizes a half-center oscillator, but gap junctions can restabilize the anti-phase pattern. J Neurophysiol 91:693-703. CrossRef Medline

Beverly M, Anbil S, Sengupta P (2011) Degeneracy and neuromodulation among thermosensory neurons contribute to robust thermosensory behaviors in Caenorhabditis elegans. J Neurosci 31:11718-11727. CrossRef Medline

Bloomfield SA, Völgyi B (2009) The diverse functional roles and regulation of neuronal gap junctions in the retina. Nat Rev Neurosci 10:495-506. CrossRef Medline

Briggman KL, Kristan WB (2008) Multifunctional pattern-generating circuits. Annu Rev Neurosci 31:271-294. CrossRef Medline

Chklovskii DB, Vitaladevuni S, Scheffer LK (2010) Semiautomated reconstruction of neural circuits using electron microscopy. Curr Opin Neurobiol 20:667-675. CrossRef Medline

Chow CC, Kopell N (2000) Dynamics of spiking neurons with electrical coupling. Neural Comput 12:1643-1678. CrossRef Medline

Curti S, Pereda AE (2004) Voltage-dependent enhancement of electrical coupling by a subthreshold sodium current. J Neurosci 24:3999-4010. CrossRef Medline

Curti S, Hoge G, Nagy JI, Pereda AE (2012) Synergy between electrical coupling and membrane properties promotes strong synchronization of neurons of the mesencephalic trigeminal nucleus. J Neurosci 32:4341-4359. CrossRef Medline

Dermietzel R (1998) Gap junction wiring: a "new" principle in cell-to-cell communication in the nervous system? Brain Res Rev 26:176-183. CrossRef Medline

Devor A, Yarom Y (2002) Electrotonic coupling in the inferior olivary nucleus revealed by simultaneous double patch recordings. J Neurophysiol 87:3048-3058. Medline

Dickinson PS, Mecsas C, Marder E (1990) Neuropeptide fusion of two motor pattern generator circuits. Nature 344:155-158. CrossRef Medline

Dugué GP, Brunel N, Hakim V, Schwartz E, Chat M, Lévesque M, Courtemanche R, Léna C, Dieudonné S (2009) Electrical coupling mediates tunable low-frequency oscillations and resonance in the cerebellar Golgi cell network. Neuron 61:126-139. CrossRef Medline

Edwards DH, Yeh SR, Krasne FB (1998) Neuronal coincidence detection by voltage-sensitive electrical synapses. Proc Natl Acad Sci U S A 95:71457150. CrossRef Medline

Evans WH, Martin PE (2002) Gap junctions: structure and function (review). Mol Membr Biol 19:121-136. CrossRef Medline

Friedman AK, Zhurov Y, Ludwar BCh, Weiss KR (2009) Motor outputs in a multitasking network: relative contributions of inputs and experiencedependent network states. J Neurophysiol 102:3711-3727. CrossRef Medline

Furshpan EJ, Potter DD (1959) Transmission at the giant motor synapses of the crayfish. J Physiol 145:289-325. Medline

Gutierrez GJ, O'Leary T, Marder E (2013) Multiple mechanisms switch an electrically coupled, synaptically inhibited neuron between competing rhythmic oscillators. Neuron 77:845-858. CrossRef Medline

Haas JS, Landisman CE (2011) State-dependent modulation of gap junction signaling by the persistent sodium current. Front Cell Neurosci 5:31. CrossRef Medline

Haas JS, Zavala B, Landisman CE (2011) Activity-dependent long-term depression of electrical synapses. Science 334:389-393. CrossRef Medline
Harris-Warrick RM, Marder E (1991) Modulation of neural networks for behavior. Annu Rev Neurosci 14:39-57. CrossRef Medline

Hooper SL, Moulins M (1989) Switching of a neuron from one network to another by sensory-induced changes in membrane properties. Science 244:1587-1589. CrossRef Medline

Hormuzdi SG, Filippov MA, Mitropoulou G, Monyer H, Bruzzone R (2004) Electrical synapses: a dynamic signaling system that shapes the activity of neuronal networks. Biochim Biophys Acta 1662:113-137. CrossRef Medline

Jang H, Kim K, Neal SJ, Macosko E, Kim D, Butcher RA, Zeiger DM, Bargmann CI, Sengupta P (2012) Neuromodulatory state and sex specify alternative behaviors through antagonistic synaptic pathways in C. elegans. Neuron 75:585-592. CrossRef Medline

Johnson BR, Peck JH, Harris-Warrick RM (1993) Amine modulation of electrical coupling in the pyloric network of the lobster stomatogastric ganglion. J Comp Physiol A 172:715-732. Medline

Kopell N, Ermentrout B (2004) Chemical and electrical synapses perform complementary roles in the synchronization of interneuronal networks. Proc Natl Acad Sci U S A 101:15482-15487. CrossRef Medline

Kopell N, Abbott LF, Soto-Trevino C (1998) On the behavior of a neural oscillator electrically coupled to a bistable element. Physica D 121: 367-395. CrossRef

Lewis TJ, Rinzel J (2000) Self-organized synchronous oscillations in a network of excitable cells coupled by gap junctions. Network 11:299-320. CrossRef Medline

Lewis TJ, Rinzel J (2003) Dynamics of spiking neurons connected by both inhibitory and electrical coupling. J Comput Neurosci 14:283-309. CrossRef Medline

Manor Y, Nadim F, Abbott LF, Marder E (1997) Temporal dynamics of graded synaptic transmission in the lobster stomatogastric ganglion. J Neurosci 17:5610-5621. Medline

Marder E (1998) Electrical synapses: beyond speed and synchrony to computation. Curr Biol 8:R795-R797. CrossRef Medline

Marder E (2012) Neuromodulation of neuronal circuits: back to the future. Neuron 76:1-11. CrossRef Medline

Marder E, Bucher D (2007) Understanding circuit dynamics using the stomatogastric nervous system of lobsters and crabs. Annu Rev Physiol 69: 291-316. CrossRef Medline

Marder E, Calabrese RL (1996) Principles of rhythmic motor pattern generation. Physiol Rev 76:687-717. Medline

McMahon DG, Brown DR (1994) Modulation of gap-junction channel gating at zebrafish retinal electrical synapses. J Neurophysiol 72:2257-2268. Medline

Morris C, Lecar H (1981) Voltage oscillations in the barnacle giant muscle fiber. Biophysical J 35:193-213. CrossRef

Muller KJ, Scott SA (1981) Transmission at a "direct" electrical connexion mediated by an interneurone in the leech. J Physiol 311:565-583. Medline

Neyton J, Trautmann A (1985) Single-channel currents of an intercellular junction. Nature 317:331-335. CrossRef Medline

Neyton J, Trautmann A (1986a) Physiological modulation of gap junction permeability. J Exp Biol 124:93-114. Medline

Neyton J, Trautmann A (1986b) Acetylcholine modulation of the conductance of intercellular junctions between rat lacrimal cells. J Physiol 377: 283-295. Medline

Nolan MF, Logan SD, Spanswick D (1999) Electrophysiological properties of electrical synapses between rat sympathetic preganglionic neurones in vitro. J Physiol 519:753-764. CrossRef

Pereda AE, Curti S, Hoge G, Cachope R, Flores CE, Rash JE (2013) Gap junction-mediated electrical transmission: regulatory mechanisms and plasticity. Biochim Biophys Acta 1828:134-146. CrossRef Medline

Pfeuty B, Mato G, Golomb D, Hansel D (2003) Electrical synapses and synchrony: the role of intrinsic currents. J Neurosci 23:6280-6294. Medline

Phelan P, Goulding LA, Tam JL, Allen MJ, Dawber RJ, Davies JA, Bacon JP (2008) Molecular mechanism of rectification at identified electrical synapses in the Drosophila giant fiber system. Curr Biol 18:1955-1960. CrossRef Medline

Piccolino M, Neyton J, Witkovsky P, Gerschenfeld HM (1982) $\gamma$-Aminobutyric acid antagonists decrease junctional communication between L-horizontal cells of the retina. Proc Natl Acad Sci US\|A 79:3671-3675. CrossRef Medline

Piccolino M, Neyton J, Gerschenfeld HM (1984) Decrease of gap junction permeability induced by dopamine and cyclic adenosine $3^{\prime}: 5^{\prime}$ - 
monophosphate in horizontal cells of turtle retina. J Neurosci 4:24772488. Medline

Prinz AA, Bucher D, Marder E (2004) Similar network activity from disparate circuit parameters. Nat Neurosci 7:1345-1352. CrossRef Medline

Rela L, Szczupak L (2003) Coactivation of motoneurons regulated by a network combining electrical and chemical synapses. J Neurosci 23: 682-692. Medline

Rela L, Szczupak L (2004) Gap junctions: their importance for the dynamics of neural circuits. Mol Neurobiol 30:341-357. CrossRef Medline

Sasaki K, Cropper EC, Weiss KR, Jing J (2013) Functional differentiation of a population of electrically coupled heterogeneous elements in a microcircuit. J Neurosci 33:93-105. CrossRef Medline

Schweighofer N, Doya K, Kawato M (1999) Electrophysiological properties of inferior olive neurons: a compartmental model. J Neurophysiol 82: 804-817. Medline

Skinner FK, Turrigiano GG, Marder E (1993) Frequency and burst duration in oscillating neurons and two-cell networks. Biol Cybern 69:375-383. CrossRef Medline

Söhl G, Maxeiner S, Willecke K (2005) Expression and functions of neuronal gap junctions. Nat Rev Neurosci 6:191-200. CrossRef Medline

Spira ME, Spray DC, Bennett MV (1976) Electrotonic coupling: effective sign reversal by inhibitory neurons. Science 194:1065-1067. CrossRef Medline

Suchyna TM, Nitsche JM, Chilton M, Harris AL, Veenstra RD, Nicholson BJ
(1999) Different ionic selectivities for connexins 26 and 32 produce rectifying gap junction channels. Biophys J 77:2968-2987. CrossRef Medline

Turrigiano G, LeMasson G, Marder E (1995) Selective regulation of current densities underlies spontaneous changes in the activity of cultured neurons. J Neurosci 15:3640-3652. Medline

Varshney LR, Chen BL, Paniagua E, Hall DH, Chklovskii DB (2011) Structural properties of the Caenorhabditis elegans neuronal network. PLoS computational biology 7:e1001066. CrossRef Medline

Veruki ML, Hartveit E (2002) AII (Rod) amacrine cells form a network of electrically coupled interneurons in the mammalian retina. Neuron 33: 935-946. CrossRef Medline

Vervaeke K, Lorincz A, Gleeson P, Farinella M, Nusser Z, Silver RA (2010) Rapid desynchronization of an electrically coupled interneuron network with sparse excitatory synaptic input. Neuron 67:435-451. CrossRef Medline

Weimann JM, Marder E (1994) Switching neurons are integral members of multiple oscillatory networks. Curr Biol 4:896-902. CrossRef Medline

White JG, Southgate E, Thomson JN, Brenner S (1986) The structure of the nervous system of the nematode Caenorhabditis elegans. Philos Trans R Soc Lond B Biol Sci 314:1-340. CrossRef Medline

White TW, Paul DL, Goodenough DA, Bruzzone R (1995) Functional analysis of selective interactions among rodent connexins. Mol Biol Cell 6:459-470. Medline 